\title{
RockOn Workshop
}

\author{
William Dirienzo, Ryan Kisiolek
}

Computer Science, Engineering, Physics, and Astronomy Department, University of Wisconsin-Sheboygan, Sheboygan, WI

\begin{abstract}
RockOn is a sounding rocket payload building workshop organized by the Colorado Space Grant Consortium, Virginia Space Grant Consortium, and the National Aeronautics and Space Administration's Wallops Flight Facility (WFF). Participants spend a week at WFF building, testing, and integrating a predesigned payload from a kit and see it launched at the end of the week. We attended the workshop with the support of the Wisconsin Space Grant Consortium and report our experience here. We recommend this program to others and plan to participate in the next level of the program, RockSat-C, this year. ${ }^{1}$
\end{abstract}

\section{Introduction}

Every year since 2008, the Colorado Space Grant Consortium (COSGC) leads the RockOn ${ }^{2}$ workshop, a hands-on workshop for students and educators with little to no experience working with sounding rockets or scientific payloads. Though a rocket plays a critical and very exciting roll, the program was not about building a rocket; it was about building what goes inside the rocket, its payload. With the cooperation of the National Aeronautics and Space Administration (NASA) and the Virginia Space Grant Consortium (VSGC), the workshop is designed to be an introduction to sounding rocket payloads and a recruiting tool for further advanced projects in the related RockSat program. Payloads from RockOn and RockSat ${ }^{3}$ are flown on a single sounding rocket at the end of the RockOn workshop.

We attended the RockOn workshop in June 2016. Our team was composed of Ryan Kisiolek, a biology and chemistry student at the University of Wisconsin-Sheboygan (UWS), William Dirienzo, Assistant Professor of Astronomy and Physics at UWS, and Christine Thompson, Assistant Director of the Wisconsin Space Grant Consortium (WSGC).

Below we share a detailed description of the program, our experiences, and plans to continue this work in the future.

\footnotetext{
${ }^{1}$ We would like to thank the RockOn and RockSat programs for a priceless learning experience and a great partner to the engineering, physics, and astronomy programs at UWS. We would like to thank the Wallops Flight Facility and NASA for accommodating the rocket programs, the Colorado and Virginia Space Grant Consortia and Chris Koehler for making the program possible. We thank the UW-Sheboygan campus and the Wisconsin Space Grant Consortium, the Computer Science, Engineering, Physics, and Astronomy Department of UW Colleges for their generous support.

${ }^{2}$ http://spacegrant.colorado.edu/national-programs/rockon-2016-home

http://spacegrant.colorado.edu/rs-c-home/previous-experiments/rs-c-2016-home
} 


\section{Wallops Flight Facility}

RockOn takes place at the Wallops Flight Facility (WFF) ${ }^{4}$, which is located about 160 miles from Washington, D.C., on Virginia's east coast, as shown in Figure 1 The WFF is primarily involved with science and exploration missions, including the support of sub-orbital research rockets, also known as sounding rockets. Their other activities include high-altitude balloons, navy operations, and launches of the Antares rockets that supply the International Space Station. The facility is split into a main base, where we lodged and where most RockOn and other WFF work takes place, and the launch base on Wallops Island, where the actual launch occurred. As part of the recruitment and outreach mission of the RockOn program, workshop participants get tours of the facility, hear about NASA internship opportunities, and have access to NASA employees across WFF operations.

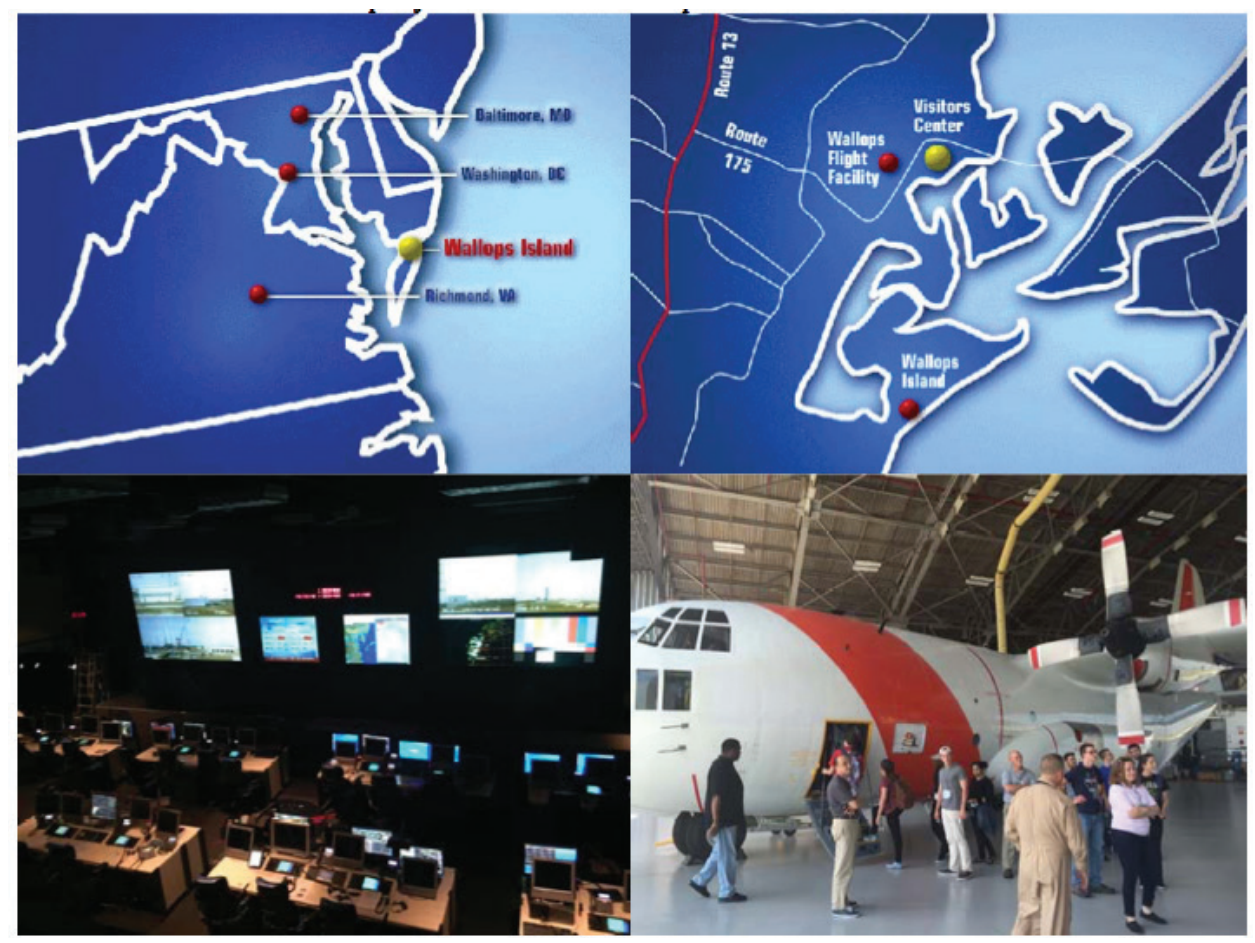

Fig. 1: Upper Left: Map of Wallops Flight Facility (WFF) on the eastern shore of Virginia. Upper Right: Map of the area around WFF and the individual bases.

Lower Left: The aircraft hangar on base.

\section{Payload Assembly \& Integration}

RockOn has a limited capacity to ensure that every participant can be on a team and fly a payload. Twenty-three teams (of three people each) build one payload from a kit designed and organized by RockOn volunteers. The program is lead by Chris Koehler from COSGC and supported by undergraduate and graduate student volunteers from previous and current RockOn and RockSat programs. After an overview of the goals of the workshop, participants' introductions, and teambuilding activities, the first few days of the workshop comprise the building of the payload. The focus starts on learning assembly techniques and shifts towards testing hardware and software. This first phase takes place in a building on the WFF main base. Each team gets their own table pre-populated with equipment, including a soldering iron, safety goggles, magnifiers, a lamp, an electrostatic discharge (ESD) mat with clips, basic tools, and the payload kit. 
Each team receives a box filled with more than seventy labeled plastic bags containing resistors, capacitors, diodes, circuit boards, and the other items needed for the payload. Teams are also supplied with binders containing the presentation slides for the assembly and testing, circuit schematics, forms for recording testing results, and part lists. These materials are also available on the RockOn website. With the close supervision and the support from the RockOn staff, participant build the payload from scratch in three days. The payloads each consist of a Geiger counter, tridirectional gyroscopes and accelerometers, pressure, temperature, and humidity sensors, an Arduino Mega microcontroller, and a microSD drive. The provided kit

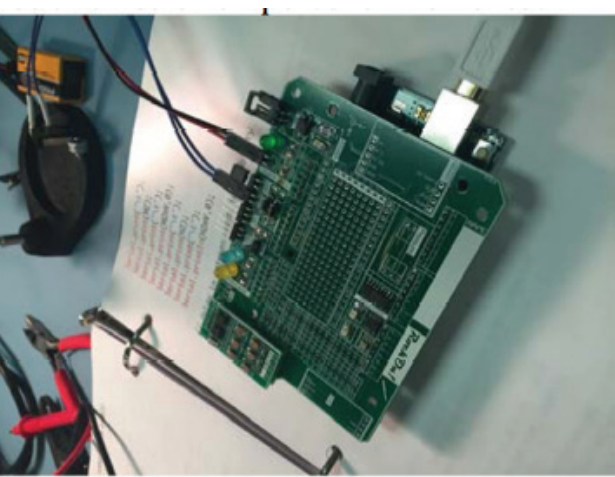

Fig. 2: The Arduino Mega with RockOn shield in an early stage of assembly and testing. includes the printed circuit boards for the Geiger counter and the shield for the Arduino that attached the other sensors and the microSD drive, shown in (Fig. 2). Teams are also allowed small personal mementos.

The assembly process is designed to be an educational experience rather than the most efficient build. Design choices are intentionally made so that participants learn different soldering techniques and use different size and shape electrical components even if they could have been avoided without comprising the functionality of the payload. The final flight code is provided to avoid errors, but participants do have to use and manipulate the testing code to gain that experience as well. Volunteers checked the progress and test results frequently. The tests include conductivity checks, LED sequences, sensor trials, and visual solder checks.

\section{Launch \& Recovery}

The RockOn and RockSat payloads were launched on the same rocket. Completed RockOn payloads sat on circular plates about a foot in diameter and were about two inches high, shown in (Fig. 3). They were aggregated into standardized canisters that were loaded onto metal racks that were then loaded directly into the rocket. The full integration and subsequent testing took a full day. Payloads received one final electrical test as they were loaded. The loaded top half of the rocket was pressurized (to keep it afloat for the later retrieval from the ocean) and underwent spin and submersion tests. Finally it was loaded onto a truck for transport to the

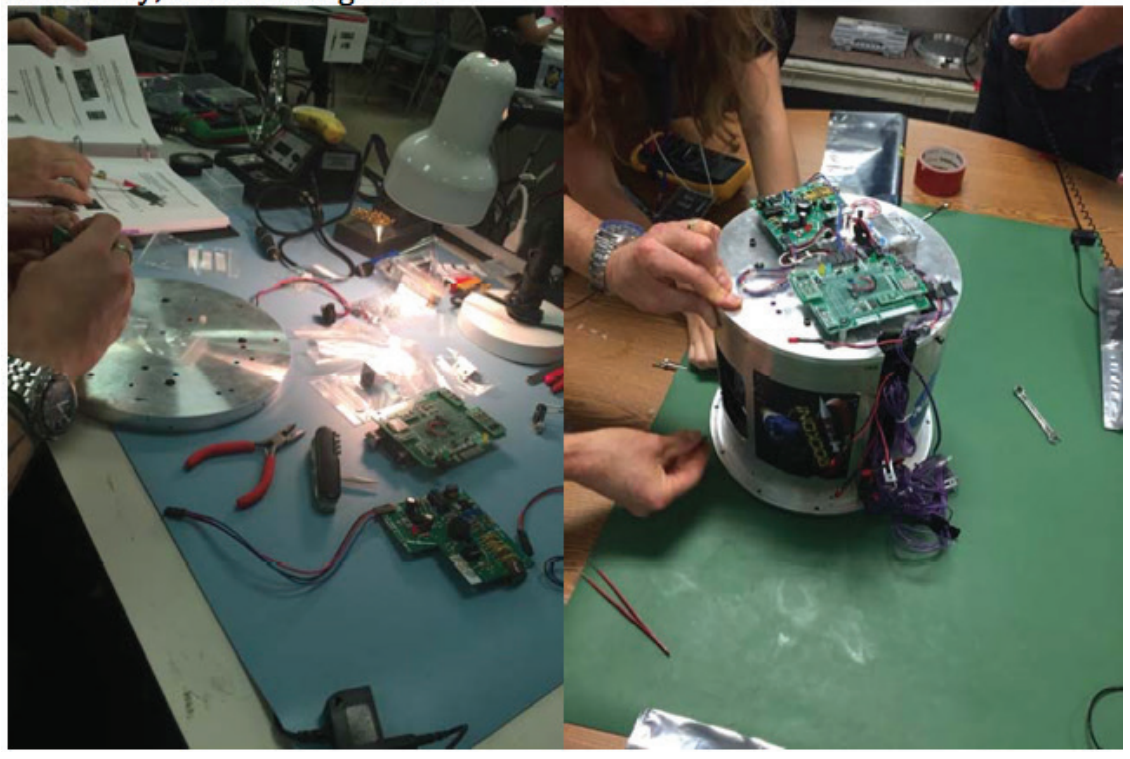

Fig. 3: Attaching our payload to the canister. 
launch facility, shown in (Fig. 4).

At the launch facility, the payload half of the rocket was loaded onto a two stage booster and slid onto a railing to guide the launch direction. It was aimed slightly east to carry it over the Atlantic Ocean for a safe recovery. For the fist time in RockOn history, the launch was delayed to the second day of the launch window due to poor weather; a (strongly) severe thunderstorm passed over Wallops on the first day; it was clear enough to launch the rocket at 6:00 AM Eastern time, the very beginning of the second day. The first stage of the booster was not securely attached to the second; the pressure of the launch kept them coupled until the first stage was spent and simply fell off the rocket before the second stage ignited. The entirety of the RockOn and RockSat program was gathered to watch the launch. The rocket rose to about 73 miles above Earth's surface, collecting data while launching, maneuvering about its axis in space for about 15 minutes, and falling back to Earth.

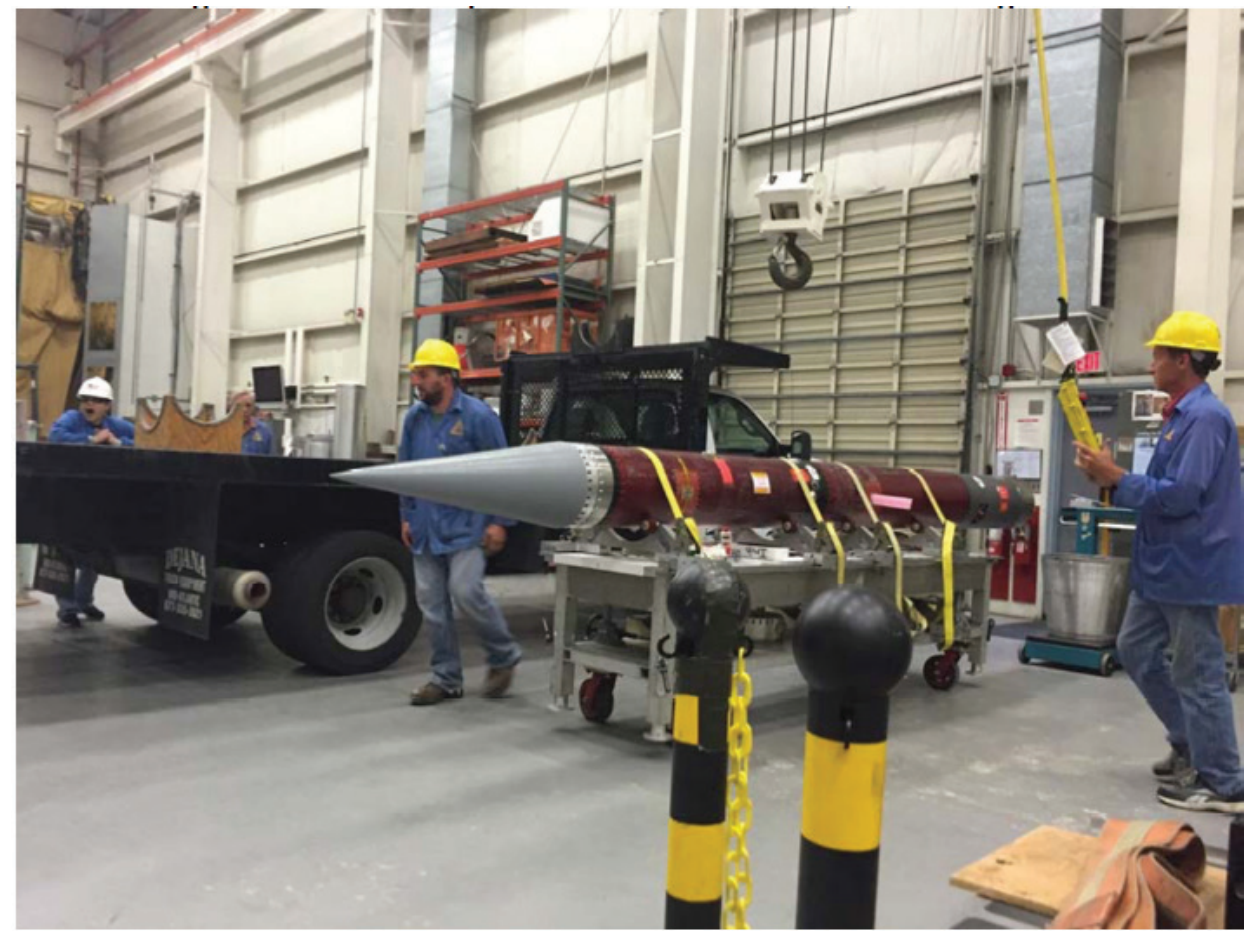

Fig. 4: The completed payload section of the rocket being loaded onto a truck to go to the launch facility.

WFF contracted a local fisherman to recover the rocket from the ocean, shown in (Fig. 5). It was brought back to harbor, loaded onto a truck, and deintegrated and WFF. Monitoring of the rocket's position via radar allowed for a very accurate determination of its landing position. RockOn teams had their payloads and data in the early afternoon the same day as the launch. 


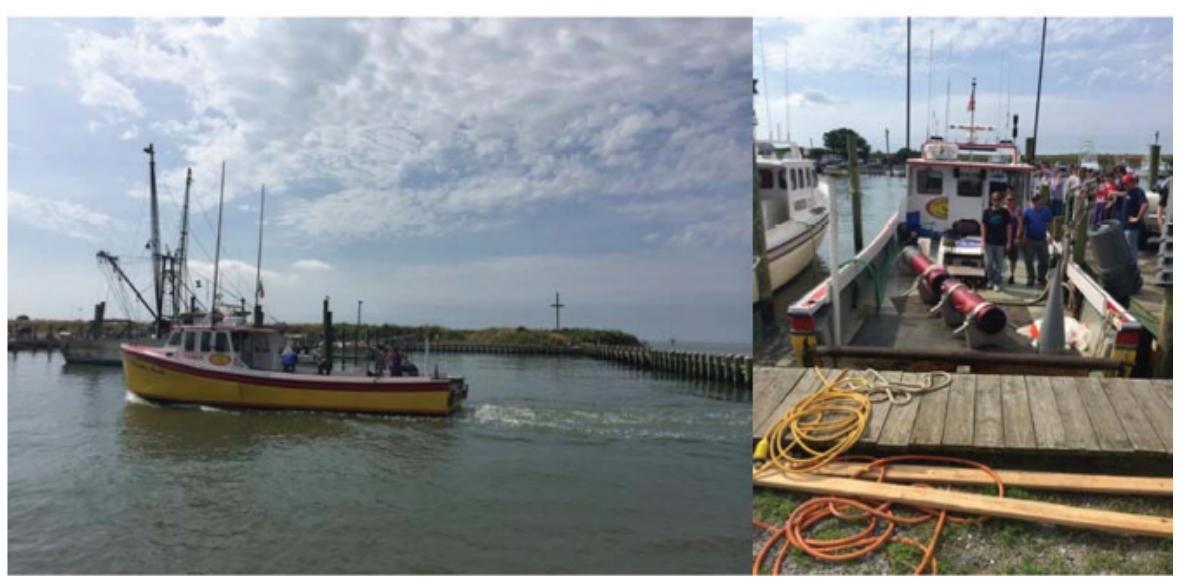

Fig. 5: Recovery of the rocket.

Our payload was a success and recorded data from launch until we removed the batteries from our payload to retrieve the data. Our sensors received only rough (if any calibration), though our data show several interesting features. The accelerometers show the two stages of the booster rockets. The gyroscopes showed a rocket initially rotating about the launch axis tumbling back to Earth after reaching apex. The temperature and pressure climbed during launch before virtually stabilizing, while the humidity also climbed during launch before falling (The pressure data revealed a dramatic fall when the rocket was opened following recovery, while the temperature and humidity showed this fall followed by significant variation as the payload was transported across the base before the batteries were disconnected).

\section{Conclusions}

The RockOn program is both inspirational and empowering. Those who take part in this program have a hands-on experience are sending something into space, and from the moment the program starts everyone is encouraged to take part in every step of the process possible. For students looking forward to a career in science this is a beautiful chance to get their hands dirty, make mistakes, learn, and move forward. This is an amazing opportunity. 

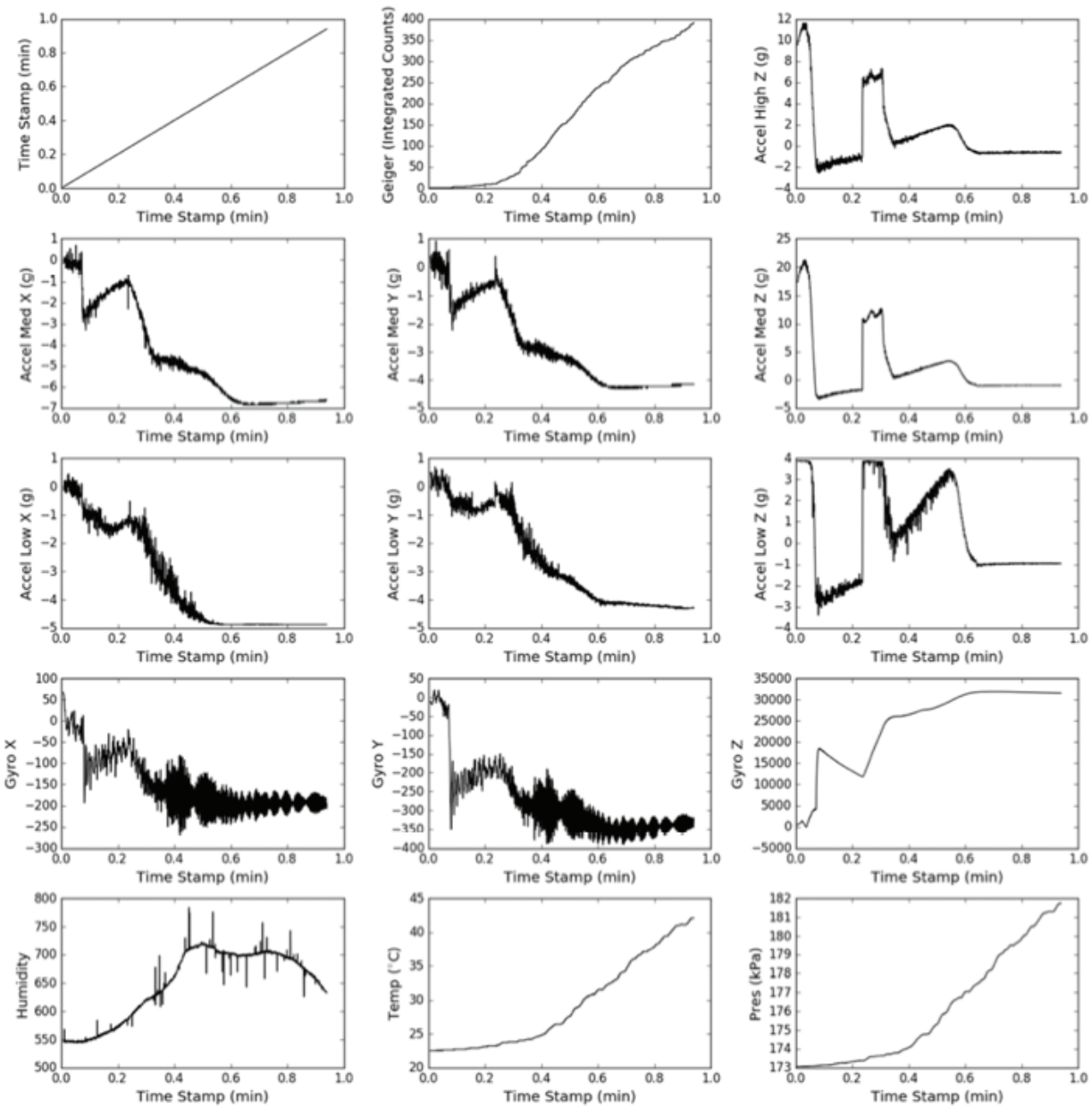

Fig. 6: Payload data from the first minute of the flight.

RockSat-C and RockSat-X are the next two levels of this sounding rocket program. In these programs the teams design an experiment, and build their own payload. These programs give students a chance to do real science while working in an aerospace setting side by side with NASA. At UWS we are currently assembling a team to do a RockSat-C project. 